Foud: I nt egr at ing Fog and $\mathrm{Cl}$ oud for 5G Enabl ed V2G Net wor ks

\begin{tabular}{|l|l|}
\hline 著者 & TAO M ng, OTA Kaor u, DONG M anxi ong \\
\hline $\begin{array}{l}\text { j our nal or } \\
\text { publ i cat } \mathrm{i} \text { on } \mathrm{t} \text { i t l e }\end{array}$ & I EEE Net work \\
\hline vol une & 31 \\
\hline number & 2 \\
\hline page $r$ ange & $8-13$ \\
\hline year & $2017-03$ 22 \\
\hline URL & ht t p: //hdl . handl e. net /10258/00009564 \\
\hline
\end{tabular}




\title{
Foud: Integrating Fog and Cloud for 5G-Enabled V2G Networks
}

\author{
Ming Tao, Member, IEEE, Kaoru Ota, Member, IEEE, Mianxiong Dong, Member, IEEE,
}

\begin{abstract}
Vehicle-to-Grid (V2G) technology is considered as a powerful approach enabling renewable energy sources to provide ancillary electrical services, and managing \& monitoring power usage in the "smart grid". However, owing to the inherent high mobility of Electric Vehicle $(E V)$, flexible and timely on-demand response services against $E V$ mobility in the V2G system must be provided. Promoted by the advantages of $5 \mathrm{G}$ communications, e.g., supporting various connectivity and faster transmission, fogs and clouds are enabled to provide realistic services, and, fog computing and cloud computing assisted V2G system in future 5G mobile network is being expected to be a new paradigm to create a new situation for V2G services. To this end, a fog- and cloud-based hybrid computing model named "Foud" applied to V2G networks in 5G is proposed. The potential V2G network services provided by "Foud" are reviewed, and some open issues and challenges for future work have been also discussed with possible solutions.
\end{abstract}

Index Terms-V2G, Cloud computing, Fog computing, 5G communications.

\section{INTRODUCTION}

Enabling renewable energy sources to provide ancillary electrical services through bi-directional communication and energy flows between $E V \mathrm{~s}$ and power grid, V2G as a critical network service in the "smart grid" is considered to be one of the most powerful approaches of managing and monitoring power usage [1]. As shown in Figure 1, to implement the power exchange and the interaction of power-related data, V2G network employs a two-way communication infrastructure, in which, power links are deployed to achieve both charging the batteries of $E V$ s by consuming power from the power grid and discharging the stored power of $E V \mathrm{~s}$ back to the power grid, and various wireless/wired communication technologies also are integrated to support the data communications between the V2G system entities (e.g., $E V$ s, Local Aggregators ( $L A G \mathrm{~s}$ ), Certification/Registration Authorities $(C A \mathrm{~s} / R A \mathrm{~s})$ and Control Centers $(C C \mathrm{~s}))$. With such a network architecture, various V2G services can be implemented [2][4], e.g., ancillary services, charging/discharging enabled energy renewable services, security and privacy related preservation services, etc..

Because $E V$ mobility is one of the unique and inherent characteristics of the V2G system, an $E V$ can perform random or purposeful movement in the V2G networks and may work in different modes accordingly. When an $E V$ is connected to a $L A G$ serving as its default access point for power and data communication, the $E V$ is working in "home mode";

Ming Tao is with Dongguan University of Technology, China

Kaoru Ota and Mianxiong Dong (mx.dong@csse.muroran-it.ac.jp) are with Muroran Institute of Technology, Japan otherwise, when an $E V$ is temporarily connected to a $L A G$ managed by a different institution, the $E V$ is working in "visiting mode" [5]. Hence, along with $E V$ mobility, new critical challenges for providing flexible and timely on-demand response V2G services must be addressed [6], [7].

Recently, cloud computing as a new computing model has been considered as a challenging research and industrial topic for many years. To on-demand achieve reliable, secure and efficient $\mathrm{V} 2 \mathrm{G}$ service responses within the implemented distributed architecture, integrating cloud computing with the V2G system can improve the service management and integration levels by enabling the V2G system entities to focus on more advanced and complicated service applications and outsourcing the basic and generic service-oriented applications to the clouds [8]. In [9], by formulating the problem of routing service request in cloud environment jointly with the analysis of power flow in the V2G system, Leon-Garcia et al. investigate the opportunities provided by cloud computing to help the V2G system on robustness and load balancing.

Although cloud computing characterized by powerful computing and storage capacities has been identified as a promising means that can offer some significant benefits for the V2G system, fog computing [10], as a new paradigm which can deliver the computing services at the edge of the cloud network and well support low-latency and location-aware services also has been demonstrated as an alternative means to ensure the desired levels to the $\mathrm{V} 2 \mathrm{G}$ services requested by moving $E V \mathrm{~s}$. In [11], based on a fog computing platform, Faruque et al. present an energy management system prototype for home and micro-grid levels with the customized control-as-services.

Expectably, under the paradigm of cloud computing and fog computing, as the increasing popularity of $E V \mathrm{~s}$ and rich $\mathrm{V} 2 \mathrm{G}$ services, both the number of accessing vehicular terminals and the volume of produced mobile data will experience tremendous growth. However, under the current architecture of mobile networks using the existing communication technologies, e.g., satellite, 3G/4G, both cloud computing and fog computing may not be perfectly suitable for ensuring the desired levels of various performance metrics to any $\mathrm{V} 2 \mathrm{G}$ services requested by moving $E V$ s. Fortunately, with the emerging of $5 \mathrm{G}$ mobile network, using the advantages of supporting various connectivity and faster transmission, more realistic services provided by various kinds of clouds and fogs are being expected to be enabled [12], [13].

However, to the best of our knowledge, optimizing the V2G network services by integrating both cloud computing and fog computing in the context of $5 \mathrm{G}$ has been investigated to a much lesser extent. To this end, a new hybrid computing model 


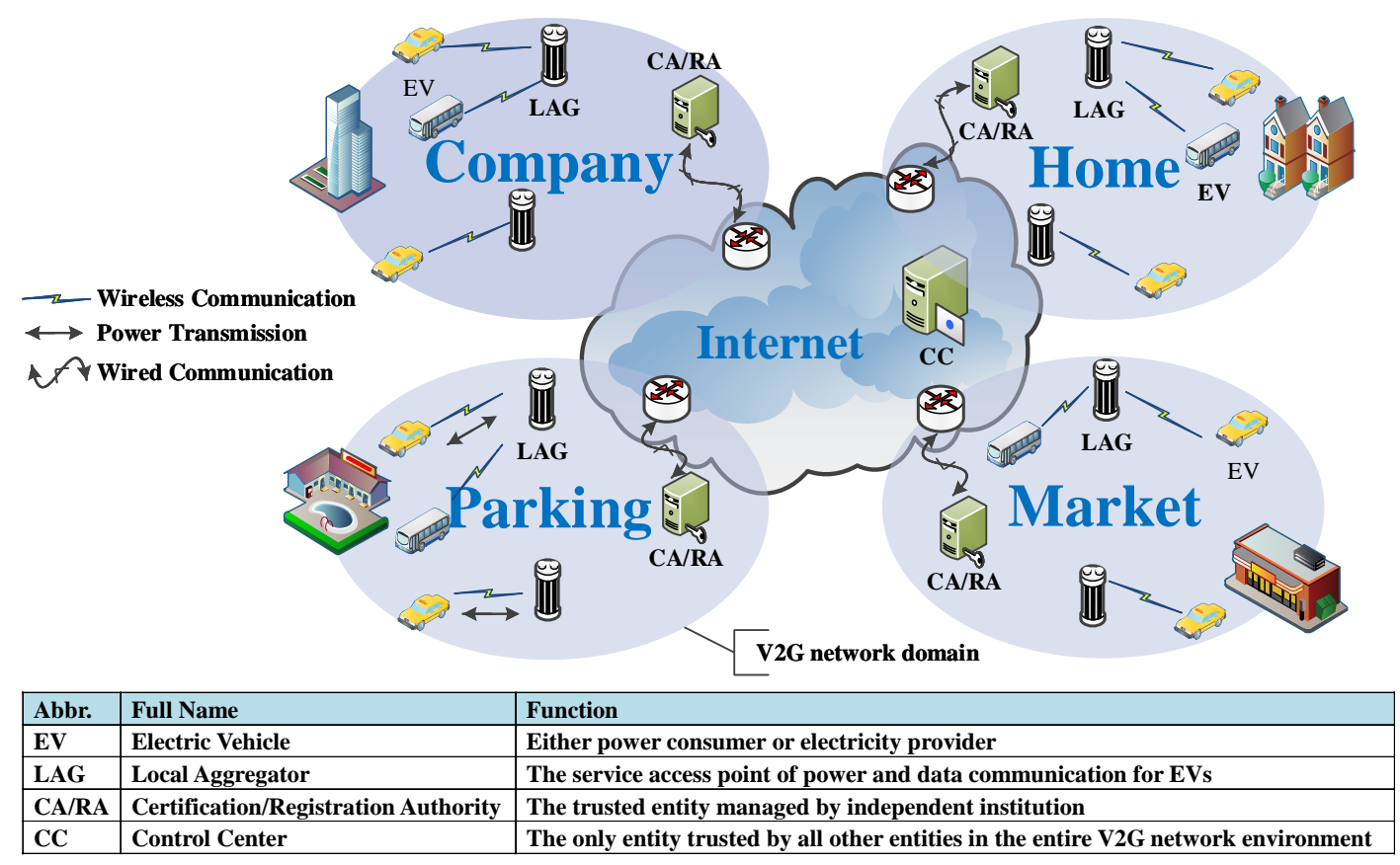

Fig. 1. An illustration of V2G network environment.

named "Foud" which makes the fog and cloud be available to the V2G system is proposed, in which, two sub-models: "Permanent Cloud" and "Temporary Fog" together constitute the infrastructure of "Foud". Different from the previous proposals, fog computing integrated into "Foud" is taken as a sub-model of "Temporary Fog" owing to the dynamic of mobile computing resources, additionally, 5G communication technologies are utilized to cope with the issue of tremendous growth of accessing vehicular terminals and produced mobile data traffic which is hard to couple with the current architecture of mobile networks using the existing communication technologies. In this article, the proposal of "Foud" is first presented, and the potential V2G services supported by "Foud" are addressed. Subsequently, the effectiveness of "Foud" is defenestrated by a simulation. Finally, considering the high mobility of $E V \mathrm{~s}$ and the dynamic of mobile computing resources, some open issues and challenges in "Foud" are discussed with possible solutions.

\section{PROPOSAL OF "FOUD"}

As shown in Figure 2, in the context of 5G, by integrating the emerging and advanced technologies of fog computing and cloud computing, a hybrid computing model for V2G networks named "Foud" is designed.

\section{A. Architecture of "Foud"}

The architecture of the proposed "Foud" is made up of two sub-models, "Permanent Cloud" and "Temporary Fog". In terms of the powerful and stationary computing and storage capacities of the conventional cloud, "Permanent Cloud" can make the virtualized computing, storage and network resources be available to the $\mathrm{V} 2 \mathrm{G}$ system entities, and mainly provides the three distinct types of services, e.g., Software-as-a-Service (SaaS), Platform-as-a-Service (PaaS) and Infrastructure-as-aService (IaaS). In "Temporary Fog", the stationary and mobile computing resources provided by the $\mathrm{V} 2 \mathrm{G}$ system entities (e.g., $L A G \mathrm{~s}, C A \mathrm{~s} / R A \mathrm{~s}, C C \mathrm{~s}$ ), on-board modules in $E V \mathrm{~s}$ and even the drivers' devices, which are located at the edge of the V2G networks are temporarily integrated together into a fog to expand the computing capacity. Accordingly, supporting different computing functions (e.g., processing, storage, networking), and integrating the $5 \mathrm{G}$ communications, "Foud" could enable the authenticated and authorized endusers in the V2G system to access all available stationary and mobile computing resources in the form of virtualization with significantly reduced costs.

Inspired by cross-layer design, "Foud" organizes the involved architecture components into three layers, User Layer, Network Layer and Service Layer. The main characteristics and functions of the three layers are respectively described as follows.

1) User Layer. The user layer composed of various kinds of end-users is the lowest-level of "Foud". In broad terms, the end-users consist of all the V2G system entities and the drivers that have the demand of using the services in "Foud". Different from the other end-users, $E V \mathrm{~s}$ and drivers have a unique feature in their inherent high mobility. Through communication and computing devices or on-installed modules, the end-users could initiate the service requests to the upper layers by service access points (SAPs). Correspondingly, the upper layers will on-demand response the service requests, and the end-users subsequently receive the service responses from the upper layers through the SAPs similarly.

2) Network Layer. The network layer made up of a variety of communication devices and technologies is designed to 


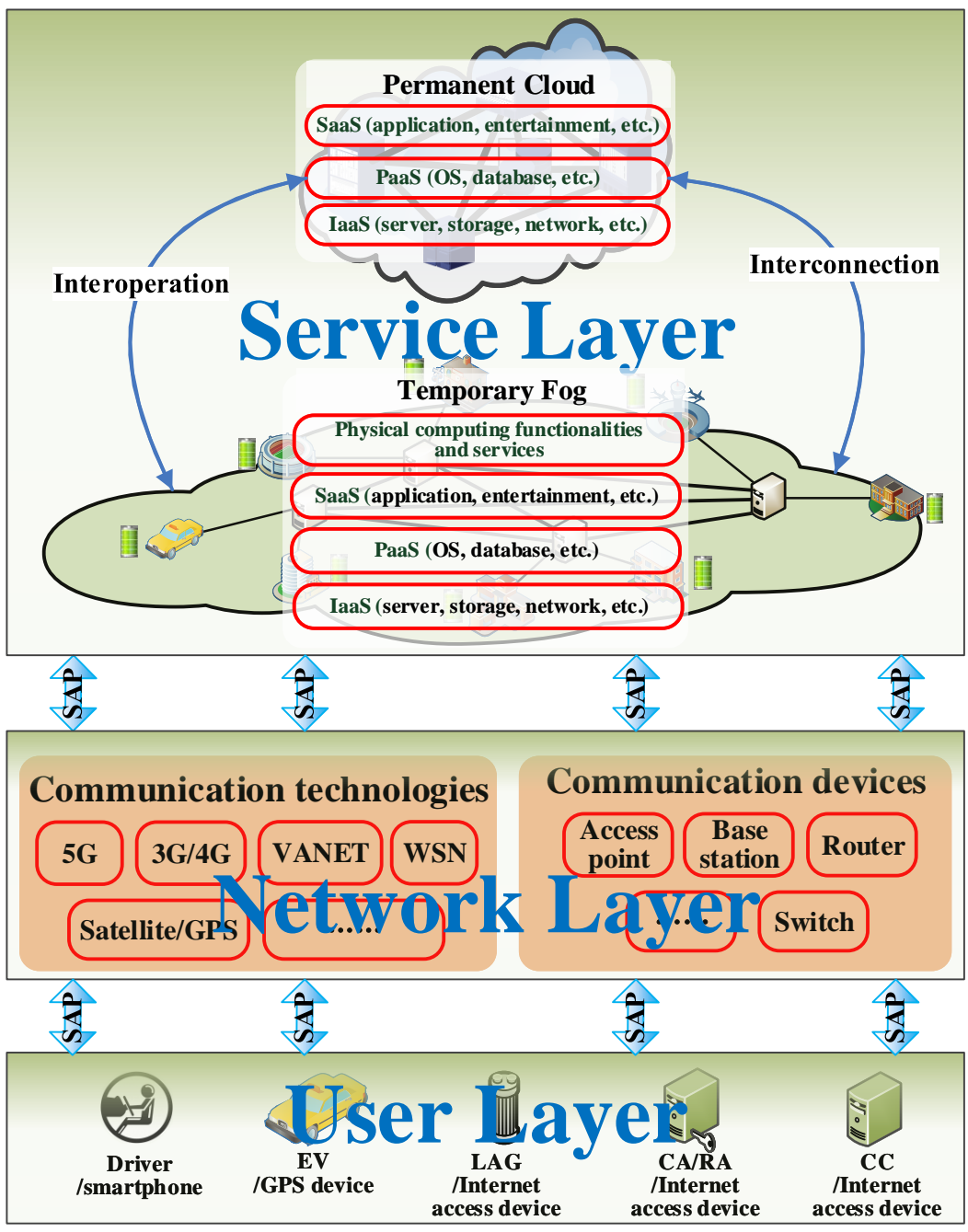

Fig. 2. Proposed "Foud" model.

implement the interactions between the end-users in the user layer and the computing resources, besides, it also used to achieve the interactions between the end-users, and the interconnection and interoperation between the defined two sublayers in the service layer. In this layer, 5G communications are the favorites, other current widely used communication technologies, e.g., 3G/4G, satellite, GPS, etc., are also integrated to form a heterogeneous convergence network. Additionally, the technology of Self-Defending Network (SDN) also can be used to shield the heterogeneity of the underlying communication facilities [14].

3) Service Layer. The service layer consists of two sublayers: a permanent layer supporting the conventional cloud and a dynamic layer supporting the temporary fog. By passing the network layer, the service layer supported by the computing capacities in "Permanent Cloud" and "Temporary Fog" could provide various kinds of V2G services for the end-users located in the user layer through SAPs.

In the permanent cloud layer, the three distinct types of services, SaaS (e.g., application, entertainment, game), PaaS (e.g., OS, database) and IaaS (e.g., server, storage, network), could be provided by various available stationary computing resources organized in the form of vitalization.

In the dynamic layer of temporary fog, under-utilized by "Permanent Cloud", a mass of computing resources on the edge of the V2G networks, e.g., the stationary computing resources provided by $L A G \mathrm{~s}, C A \mathrm{~s} / R A \mathrm{~s}, C C \mathrm{~s}, E V \mathrm{~s}$ and drivers at the rest state, and the mobile computing resources provided by $E V \mathrm{~s}$ and drivers in the moving state, are integrated to form the infrastructure of fog computing. Due to the $E V$ and driver mobility, the mobile computing resources provided by them are temporarily connected to the fog, accordingly, this sub-model in "Foud" is named as "Temporary Fog" which is different from the work in the previous proposals. Additionally, because fog computing is based on the service computing architecture model of para-virtualization, physical computing functionalities and services, and virtualized services in the form of SaaS, PaaS and IaaS, both could be made available to the end-users in the user layer through SAPs.

\section{B. Interconnection and Interoperation Between Sub-Models}

As stated above, the two sub-models, "Permanent Cloud" and "Temporary Tog" together make up the architecture model 
of "Foud". The interconnection between the two sub-models can be achieved by a global network consisting of all computing resources in the service layer through the introduced internetworking technologies in the network layer. In addition, to uniformly manage and control the merged network in "Foud", different network techniques and protocols must be supported.

Based on the enabled interconnection, the issue of interoperability between the two sub-models could be addressed as well. Through the network, protocol, interface and security (e.g., certification and authorization) techniques to achieve a global control for the V2G system, "Permanent Cloud" and "Temporary Fog" could be fully cooperated and interoperable to perform the joint implementation of V2G services without any additional costs.

\section{Potential V2G Services and Applications}

Making full use of the advantages of fog computing, cloud computing and 5G communications, "Foud" could facilitate the quick growth of enriched V2G services of the following partial summarized applications.

Powerful computing and standardized data storage: With the powerful computing capacity in "Permanent Cloud" and the expanded computing capacity provided by "Temporary Fog", "Foud" could provide compute-intensive services and applications, e.g., planning the shortest path between the power-thirsting $E V \mathrm{~s}$ and charging stations, optimizing the deployment of charging stations to provide high quality services with respect to usability, security and low latency. Additionally, a volume of sensed and aggregated data, e.g., traffic, movement of $E V \mathrm{~s}$, battery depletion state, geographic locations of recharging stations, could be stored in "Foud" with standardized data formats.

Balanced power management services and applications: Power management is a major concern in the "smart grid". By utilizing the powerful computing and storage capacities in "Foud" to aggregate and analyze the massive data information, e.g., the cycles of $E V$ power demand and renewable generation integration, the power consumption prediction of $E V$, "Foud" could primely enable the balanced power management services and applications by reasonable supply of power and providing flexible on-demand response services for the $E V \mathrm{~s}$. In addition, interestingly, because $E V$ s could flexibly act as both energy storages and energy transporters, balanced power management among different districts also could be achieved through $E V$ mobility.

Resource-sensitive services and applications: With the advantage of the significantly faster transmission performance of 5G communication technologies integrated in "Foud", in terms of high bandwidth and low latency, "Foud" is expected to be available for providing resource-limited endusers (e.g., moving $E V$ s) with resource-sensitive services and applications, e.g., in-vehicle infotainment, traffic accident notification, collision avoidance, rescuing services needed by power-depleted $E V$ s.

Location-aware services and applications: With the cooperation of the architecture components in "Foud", "Foud" could provide the end-users with various location-aware services and applications, e.g., help to discover geographic locations and real-time information of available recharging stations based on the $E V$ mobility and battery depletion state to avoid imbalance using the recharging stations and prolonging the waiting time.

Business services and applications: Promoted by the benefit on mobile big data delivery using $5 \mathrm{G}$ communications, and fog- and cloud-assisted computing, "Foud" is made be available to rich business services and applications. For example, using the shared sensed real-time power usage and pricing information, the power supplier could re-design the power grid around the recharging stations, schedule their provisioning around the clock, and implement dynamic pricing during peakhours and non-peak hours, the customers also could earn lucrative economic benefit from these real-time information.

\section{Simulation And Results}

In this section, we set up a simulation using OMNeT++ to qualitative analyze and evaluate the effectiveness of "Foud". In the simulation, using a Quadtree communication structure, we design a prototype which consists of one cloud computing node as root, 4 fog computing nodes as leaves, 500 randomly moving $E V \mathrm{~s}$ on the road network (an urban area covers $5.0 \times 5.0 \mathrm{~km}$ ). The entire area is uniformly divided into four parts, each part is managed by a fog node which can directly communicate with the inside $E V$ s, and each deployed randomly moving $E V \mathrm{~s}$ initiates a $\mathrm{V} 2 \mathrm{G}$ service request. To ensure the $\mathrm{V} 2 \mathrm{G}$ service quality experienced by the $E V \mathrm{~s}$, the response time defined as the maximum time interval from submitting the service request to receiving the response is significant for real time. Accordingly, the effectiveness of "Foud" is measured with respect to the response time of V2G service request in this work. Additionally, in the simulation, to reflect the advantages of future $5 \mathrm{G}$ communication technologies, we consider the test network in two situations: i) the network without any loads, and ii) the network with loads $(512 \mathrm{kbps})$, to approximatively indicate the transmission performance difference between future $5 \mathrm{G}$ communication technologies and existing communication technologies, and observe the differences in the response times in the two different network situations.

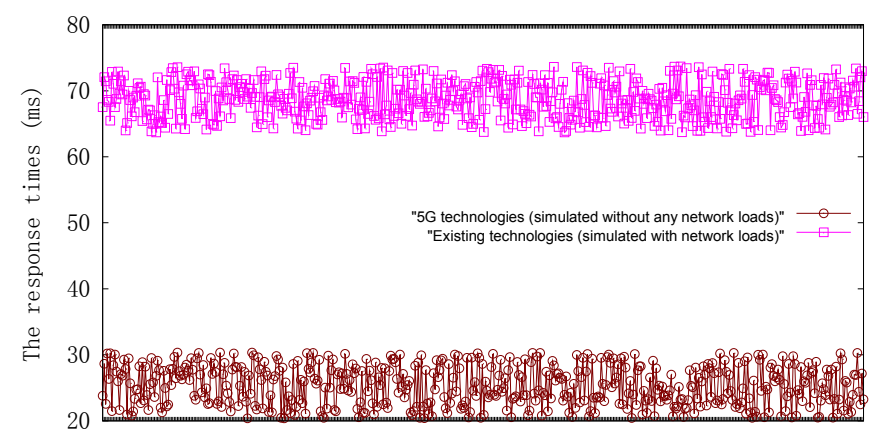

500 V2G service requests initiated by randomly moving EVs

Fig. 3. The response times in two different network situations. 
The comparison for the response times of $\mathrm{V} 2 \mathrm{G}$ service requests initiated by 500 randomly moving $E V \mathrm{~s}$ in the two different network situations is shown in Figure 3. The further analysis results of the response times are shown in Table I. From Table I, we can clearly see that, constrained by the current architecture of mobile networks using the existing communication technologies, transmitting a volume of mobile data will cause serious transmission delay which consequently brings about adversely affect on the response times of $\mathrm{V} 2 \mathrm{G}$ service requests. In comparison, with the significantly faster transmission performance of $5 \mathrm{G}$ communications, the response times of $\mathrm{V} 2 \mathrm{G}$ service requests are more acceptable.

TABLE I

COMPARISONS FOR THE RESPONSE TIMES.

\begin{tabular}{c|c|c}
\hline $\begin{array}{c}\text { Average response time } \\
(\mathrm{ms})\end{array}$ & $\begin{array}{c}5 \mathrm{G} \text { technologies } \\
\text { (Simulated without any network loads) }\end{array}$ & 25.36 \\
\cline { 2 - 3 } & $\begin{array}{c}\text { Existing technologies } \\
\text { (Simulated with network loads) }\end{array}$ & 68.63 \\
\hline $\begin{array}{c}\text { Standard deviation } \\
(\mathrm{ms})\end{array}$ & $\begin{array}{c}5 \mathrm{G} \text { technologies } \\
\text { (Simulated without any network loads) }\end{array}$ & 7.88 \\
\cline { 2 - 3 } & $\begin{array}{c}\text { Existing technologies } \\
\text { (Simulated with network loads) }\end{array}$ & 8.92 \\
\hline
\end{tabular}

\section{OPEn Issues ANd Challenges}

New architectures and platforms for V2G networks, such as "Foud" proposed in this work, must be provably efficient, scalable, secure and reliable before starting their large-scale deployment. Existing mechanisms and approaches, such as the mentioned $5 \mathrm{G}$ communications, fog computing and cloud computing, however, are not yet fully satisfactory in meeting all these requirements at the same time, especially, under $E V$ mobility and dynamic participation of mobile computing resources. There are still some significant open issues and challenges that should be addressed as the directions in future work.

Green "Foud": Energy efficient as one of the major concerns in conventional cloud computing has been investigating as a challenging academical and industrial topic for many years. In "Foud", although the redistribution of energy consumption could be achieved by extendedly utilizing the mobile computing resources in "Temporary Fog", instead of performing the centralized energy consumption in "Permanent Cloud", novel energy-efficient solutions for green "Foud" by cooperating "Permanent Cloud" and "Temporary Fog", e.g., reducing the energy consumption caused by under-utilized resources which accounts for abundant actual energy use, still remain critical challenges. Additionally, due to $E V$ mobility, some new open issues of using mobile computing resources have been arisen as well, e.g., when a mobile computing device leaves its current access network, the maintained mobile communication link will be lost, excessive transactions of re-searching and re-link routing will cause serious energy consumption, hence, an efficient mechanism of updating the information of mobile communication link loss with reduced energy cost should be investigated.

Cooperative resource management in "Foud": Due to $E V$ mobility, the computing resources provided by mobile computing devices are integrated into "Temporary Fog" in a dynamic manner. Therefore, the performance of V2G services and applications in "Foud" is seriously influenced by $E V$ mobility, efficient resource management by optimally dynamic integrating mobile computing resources into "Temporary Fog" and cooperating "Permanent Cloud" and "Temporary Fog" as an important open issue that also needs to be addressed accordingly.

Persistence of perceived QoS: "Foud" is greatly expected to be available to various V2G services and applications for the end-users in terms of ensuring the desired level of quality of service (QoS). However, due to $E V$ mobility and the dynamic participation of mobile computing resources in "Temporary Fog", providing on-demand response services with availability, reliability, scalability, adaptability, sustainability and performance for moving $E V$ s still remains a critical challenge. Consequently, developing efficient mechanisms to achieve mobility management for $E V$ s and maintain the service execution conditions by dynamically integrating the mobile computing resources without being influenced by the dynamic mobile computing resources could persistently ensure the desired level of perceived QoS.

Security and privacy preservation: V2G networks as highly distributed systems are different from other broadly applied communication systems, especially, in which, the employed two-way communication infrastructure not only facilitate the functionalities for data and power delivery, but also facilitate cyber-physical attacks, hence, the problem of security and privacy preservation in V2G networks maybe more severe [15]. Although there are many proposals have been proposed to address this severe problem, these involved open issues, e.g., anonymous mutual authentication, session key establishment, location and identification related privacy preservation, privacy-preserving based charging and discharging, billing and payment, and data publication, still remain the critical challenges in "Foud" due to the inherent high mobility of $E V \mathrm{~s}$ and utilizing the mobile computing resources in "Temporary Fog" as services. Consequently, cooperating the advantages of the componential technologies used in "Foud" to ensure security and privacy should be investigated in the future.

\section{CONCLUSION}

A new hybrid computing model for $\mathrm{V} 2 \mathrm{G}$ networks named "Foud" is proposed in this article. The proposal of "Foud" made up of two sub-models of "Permanent Cloud" and "Temporary Fog" organizes the involved architecture components into three layers. The user layer is composed of various kinds of end-users in the V2G system, and the service layer divided into two sub-layers: permanent cloud layer and temporary fog layer, provides various kinds of $\mathrm{V} 2 \mathrm{G}$ services for the user layer. To ensure the interaction and interconnection between the "Foud" components, the network layer taking 5G communications as the favorites is designed as well. Making full use of the advantages of the mentioned advanced technologies, "Foud" could facilitate the quick growth of enriched V2G services and applications. However, due to the inherent high 
mobility of $E V \mathrm{~s}$ and the dynamic participation of mobile computing resources, there are still some significant open issues and challenges that should be addressed in the future.

\section{Acknowledgement}

This work is partially supported by the National Natural Science Fund, China (Grant No. 61300198); the Guangdong University Scientific Innovation Project (Grant No. 2013KJCX0177 \& No. 2014KTSCX188); the outstanding young teacher training program of the Education Department of Guangdong Province (Grant No. YQ2015158); Guangdong Provincial Science \& Technology Plan Projects (Grant No. 2016A010101035); and JSPS KAKENHI (Grant No. 16K00117 \& No. 15K15976 \& No. 26730056), JSPS A3 Foresight Program. Mianxiong Dong is the corresponding author for this paper.

\section{REFERENCES}

[1] X. Fang, S. Misra, G. Xue, and et al., "Smart Grid-The New and Improved Power Grid: A Survey," IEEE Communications Surveys \& Tutorials, vol. 14, no. 4, pp. 944-980, 2012.

[2] V. C. Gungor, D. Sahin, and T. Kocak, "A survey on smart grid potential applications and communication requirements," IEEE Transactions on Industrial Informatics, vol. 9, no. 1, pp. 28-42, 2013.

[3] Z. M. Fadlullah, M. M. Fouda, N. Kato, and et al., "Toward Intelligent Machine-to-Machine Communications in Smart Grid," IEEE Communications Magazine, vol. 49, no. 4, pp. 60-65, 2011.

[4] M. M. Fouda, Z. M. Fadlullah, N. Kato, and et al., "A lightweight message authentication scheme for smart grid communications," IEEE Transactions on Smart Grid, vol. 2, no. 4, pp. 675-685, 2011.

[5] Y. Zhang, S. Gjessing, H. Liu, H. Ning, L. T. Yang, and M. Guizani, "Securing vehicle-to-grid communications in the smart grid," IEEE Wireless Communications, vol. 20, no. 6, pp. 66-73, 2013.

[6] X. Tan, Y. Wu, and D. H. K. Tsang, "Optimal energy trading with battery energy storage under dynamic pricing," in IEEE International Conference on Smart Grid Communications (SmartGridComm). IEEE, 2014, pp. 722-727.

[7] L. Guo, M. Dong, K. Ota, and et al., "Event-Oriented Dynamic Security Service for Demand Response in Smart Grid employing Mobile Networks," China Communications, vol. 12, no. 12, pp. 63-75, 2015.

[8] S. Bera, S. Misra, and J. J. P. C. Rodrigues, "Cloud computing applications for smart grid: A survey," IEEE Transactions on Parallel and Distributed Systems, vol. 26, no. 5, pp. 1477-1494, 2015.

[9] A. H. Mohsenian-Rad and A. Leon-Garcia, "Coordination of Cloud Computing and Smart Power Grids," in IEEE International Conference on Smart Grid Communications (SmartGridComm). IEEE, 2010, pp. 368-372.

[10] I. Stojmenovic and S. Wen, "The Fog computing paradigm: Scenarios and security issues," in Federated Conference on Computer Science and Information Systems (FedCSIS). IEEE, 2014, pp. 1-8.

[11] M. A. A. Faruque and K. Vatanparvar, "Energy Management-as-aService Over Fog Computing Platform," IEEE Internet of Things Journal, vol. 3, no. 2, pp. 161-169, 2016.

[12] D. Wubben, P. Rost, and J. S. Bartelt, "Benefits and impact of cloud computing on $5 \mathrm{G}$ signal processing: Flexible centralization through cloud-RAN," IEEE Signal Processing Magazine, vol. 31, no. 6, pp. 3544, 2014.

[13] S. Kitanov, E. Monteiro, and T. Janevski, "5G and the Fog-Survey of related technologies and research directions," in 18th Mediterranean Electrotechnical Conference (MELECON). IEEE, 2016, pp. 1-6.

[14] M. Dong, H. Li, K. Ota, and et al., "Rule Caching in SDN-Enabled Mobile Access Networks," IEEE Network, vol. 29, no. 4, pp. 40-45, 2015.

[15] W. Han and Y. Xiao, "Privacy preservation for V2G networks in smart grid: A survey," Computer Communications, vol. 91-92, pp. 17-28, 2016.

Biographies

Ming Tao is currently an associate researcher at the School of Computer Science and Network Security of Dongguan
University of Technology and the Director of the Key Laboratory of the Wireless Sensor Network System of Dongguan. He received his B.S. degree in computer science and technology from Anhui University, Hefei, China, in 2007 and M.S. and Ph.D. degrees in computer application technology from the South China University of Technology, Guangzhou, China, in 2009 and 2012, respectively. His primary research interests include protocol design and performance analysis in next-generation wireless/mobile networks, high performance computing and grid technology. He has served as a reviewer for several IEEE international conferences and international journals.

Kaoru Ota is currently an Assistant Professor with the Department of Information and Electronic Engineering, Muroran Institute of Technology, Japan. She received her M.S. degree in Computer Science from Oklahoma State University, USA in 2008 and B.S. and Ph.D. degrees in Computer Science and Engineering from the University of Aizu, Japan in 2006 and 2012, respectively. She serves as an editor for Peer-to-Peer Networking and Applications (Springer), Ad Hoc \& Sensor Wireless Networks and International Journal of Embedded Systems (Inderscience) as well as a guest editor for IEEE Wireless Communications and IEICE Transactions on Information and Systems. Her research interests include wireless networks, cloud computing, and cyber-physical systems.

Mianxiong Dong is currently an Associate Professor in the Department of Information and Electronic Engineering at the Muroran Institute of Technology, Japan. He received his B.S., M.S., and Ph.D. degrees in computer science and engineering from the University of Aizu, Aizuwakamatsu, Japan, in 2006, 2008, and 2013 respectively. He serves as an Editor for IEEE Communications Surveys and Tutorials, IEEE Network, IEEE Wireless Communications Letters, IEEE Cloud Computing, IEEE Access, and Cyber-Physical Systems (Taylor \& Francis) as well as a leading guest editor for ACM Transactions on Multimedia Computing, Communications and Applications (TOMM), IEEE Transactions on Emerging Topics in Computing (TETC), and IEEE Transactions on Computational Social Systems (TCSS). His research interests include wireless networks, cloud computing, and cyber-physical Systems. 